\title{
A constitutive model for tissue adaptation: Necrosis and stress driven growth
}

\author{
F.J. Bellomo ${ }^{\mathrm{a}, \mathrm{c}, *}$, F. Armero ${ }^{\mathrm{b}}$, L.G. Nallim ${ }^{\mathrm{a}, \mathrm{c}}$, S. Oller ${ }^{\mathrm{c}}$ \\ a INIQUI (CONICET), Faculty of Engineering, National University of Salta, Av. Bolivia 5150, 4400 Salta, Argentina ${ }^{1}$ \\ ${ }^{\mathrm{b}}$ Department of Civil and Environmental Engineering, University of California, Berkeley, CA 94720, United States ${ }^{2}$

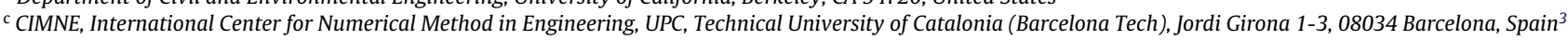

\section{A R T I C L E I N F O}

\section{Article history:}

Received 7 August 2011

Received in revised form 26 October 2011

Available online 23 November 2011

\section{Keywords:}

Soft tissue necrosis

Stress driven growth

Rule of mixtures

\begin{abstract}
A B S T R A C T
This paper presents a general constitutive model for tissue stress driven growth and necrotic remodeling. The general theoretical framework is given by a kinematics formulation in large strain. The model allows the analysis of tissue behaviour considering the coupling of biological and mechanical fields, through the implementation of an internal variable that represents the bioavailability, together with the growth induced by mechanical stimuli. To take into account the tissue necrosis phenomenon a reinterpretation of the classical theory of mixtures is proposed. The proposed formulation is based on the consideration of two components, normal tissue and necrotic tissue, each one with its own volumetric participation, which is treated in an evolutive way, allowing simulating the phenomena of ischemia. This article also presents a brief description of the numerical implementation of the model as well as the obtained results, which shows the process of necrosis of part of the tissue, its influence on the surrounding healthy tissue, and the interaction between the nutrients availability and the stress driven growth and necrotic remodeling process.
\end{abstract}

(C) 2011 Elsevier Ltd. All rights reserved.

\section{Introduction}

Tissues require a constant blood supply to obtain nutrients, oxygen and to eliminate the metabolic wastes. Ischemia is an absolute or relative shortage of the blood supply to a tissue resulting in damage because of the lack of oxygen, nutrients and the buildup of metabolic wastes. The study of this process is important because it is related with several frequent pathologies: atherosclerosis, hypoglycemia, tachycardia, hypotension, thromboembolism, outside compression of a blood vessel, embolism, localized extreme cold, arteriovenous malformations, and peripheral artery occlusive disease.

Acute ischemia causes tissue necrosis generating stiffer nonactive scar tissue. The mechanical properties of the scar are an important factor in the determination of the heart function after an acute ischemic episode (Fomovsky and Holmes, 2010).

The analysis of growth and remodeling using mixture has been addressed by several researchers, i.e. Humphrey and Rajagopal (2002), Ateshian (2009) and Ambrosi et al. (2010). In this paper

\footnotetext{
* Corresponding author at: Facultad de Ingeniería, Universidad Nacional de Salta, Av. Bolivia 5150, 4400 Salta, Argentina. Tel.: +54387 4258615; fax: +543874255410. E-mail addresses: facundobellomo@yahoo.com.ar, facundobellomo@conicet.gov.ar(F.J.Bellomo), armero@ce.berkeley.edu(F.Armero), lnallim@unsa.edu.ar (L.G. Nallim), sergio.oller@upc.edu (S. Oller).

1 http://www.ing.unsa.edu.ar/.

2 http://www.ce.berkeley.edu/.

3 http://www.cimne.com/.
}

a constitutive model to simulate the necrotic remodeling phenomena is proposed. The model includes three fundamental concepts: the biological and mechanical fields, a two-phase continuum theory and an approach of the evolution of the volumetric participation of the scar tissue. The paper is divided into sections according to the statement above mentioned. Finally, numerical results that show the validity, efficiency and potentialities of the proposed formulation are presented. Soft tissues exhibit a quasi-incompressible behaviour, the incompressibility treatment is addressed following Appendix A.

\section{Constitutive stress driven growth/atrophy model considering biological availability}

A detailed analysis regarding the theoretical aspects of growth can be found in Taber (2009), Epstein and Maugin (2000) and Ambrosi and Guana (2007).

We present in this section a brief summary of the constitutive model of mechanical-biological growth in soft tissues proposed in Oller et al. (2010), along the lines of the formulations proposed in Rodriguez et al. (1994), Lubarda and Hoger (2002), Himpel et al. (2005), Kuhl et al. (2007), Kroon et al. (2009) and Goktepe et al. (2010), among others.

We consider finite deformation kinematics, with the deformation of the solid $\mathcal{B} \subset \mathbb{R}^{3}$ defined by a one-to-one map $\varphi: \mathcal{B} \rightarrow \mathbb{R}^{3}$ giving the current positions $x=\varphi(X) \in \mathbb{R}^{3}$ of the material particles $X \in \mathcal{B}$. In this context, growth is modeled by the multiplicative 
decomposition of the deformation gradient $\boldsymbol{F}=\partial \boldsymbol{x} / \partial \boldsymbol{X}$ (Lubarda and Hoger, 2002)

$\boldsymbol{F}=\boldsymbol{F}^{e} \cdot \boldsymbol{F}^{g}$

for an elastic part $\boldsymbol{F}^{e}$ and an incompatible part $\boldsymbol{F}^{g}$ that includes the effects of growth and atrophy.

The elastic part of the deformation gradient $\boldsymbol{F}^{e}$ defines the mechanical response of the tissue. In the case considered in this paper of a purely elastic response for the bulk tissue, this definition is done through an elastic potential $W\left(\rho_{0}, \hat{\boldsymbol{C}}\right)$ in terms of the right Cauchy-Green tensor $\hat{\boldsymbol{C}}=\boldsymbol{F}^{e^{T}} \cdot \boldsymbol{F}^{e}$ (following classical arguments based on frame indifference of the resulting model) and the reference density $\rho_{0}$, which evolves to model (isotropic) growth as discussed below. In this setting, the second Piola-Kirchhoff stress tensor $\boldsymbol{S}$ in the reference configuration is obtained as

$\boldsymbol{S}=\boldsymbol{F}^{g^{-1}} \cdot \hat{\boldsymbol{S}} \cdot \boldsymbol{F}^{g^{-T}}$ for $\hat{\boldsymbol{S}}=2 \rho_{0} \frac{\partial W\left(\rho_{0} \hat{\boldsymbol{C}}\right)}{\partial \hat{\boldsymbol{C}}}$

with $\hat{\boldsymbol{S}}$ corresponding to the second Piola-Kirchhoff stress tensor in the intermediate configuration defined by the local mapping $\boldsymbol{F}^{g}$. Note again that the reference density $\rho_{0}$ evolves due to the growth, but the partial derivative appearing in Eq. (2) involves a fixed value of this density. Simple algebraic manipulations lead also to the relation

$$
\text { for } \boldsymbol{C}=\boldsymbol{F}^{T} \cdot \boldsymbol{F}=\boldsymbol{F}^{g^{T}} \cdot \hat{\boldsymbol{C}} \cdot \boldsymbol{F}^{g}
$$

the total right Cauchy-Green tensor $\boldsymbol{C}$. The Cauchy stresses $\sigma$ are then given by in terms of the rate of the isotropic growth stretch $\dot{\vartheta}$. We note that given Eq. (8) relations (2) and (3) reduce in the isotropic growth under consideration to

$\boldsymbol{S}=\frac{1}{\vartheta^{2}} \hat{\boldsymbol{S}}$ and $\boldsymbol{C}=\vartheta^{2} \hat{\boldsymbol{C}}$

respectively (so, by the way, $\boldsymbol{S}: \boldsymbol{C}=\hat{\boldsymbol{S}}: \boldsymbol{C}$ ).

Typical models of growth consider only that this growth rate is controlled by a stress stimulus. However, a simple mechanical stimulus is not enough, since mass increases require that the metabolism is able to allow the growth of the tissue. To accommodate these metabolic conditions, Oller et al. (2010) proposed an internal variable to take into account the biological availability for growth (named $\theta$ ) related as discussed below to the presence or absence of nutrients controlling the growth. In this way, the evolution rule

$\dot{\vartheta}=g\left(\operatorname{tr} \sigma, \sigma_{e q}^{*}\right) f(\theta, \vartheta)$

is proposed, where the function $g\left(\operatorname{tr} \sigma, \sigma_{e q}^{*}\right)$ models the mechanical stimulus for growth/atrophy in terms of the stress $\sigma$ (in terms of its trace tr $\sigma$ and a stress parameter $\sigma_{e q}^{*}$ to be defined below), and the function $f(\theta, \vartheta)$ regulates the metabolic part of the growth according with the aforementioned biological availability to generate new tissue. The bioavailability internal variable $\theta$ represents the volume of new tissue $d v^{g}$ that can be generated by the metabolism with the available nutrients referred to the initial tissue volume. The expression of this internal variable is given by:

$\sigma=\frac{1}{J} \boldsymbol{F} \cdot \boldsymbol{S} \cdot \boldsymbol{F}^{T}=\frac{1}{J} \boldsymbol{F}^{e} \cdot \hat{\boldsymbol{S}} \cdot \boldsymbol{F}^{e}$

defined in the current configur to

To characterize the growth under the deformation $\varphi$ $\left(J^{e} J^{g}\right) d V$ between the referen tial (deformed) volume el $J^{g}=$ det $F^{g}$. Growth or atrophy is constant density. Denoting by $\rho_{0}^{i n i}$, we then have the relation

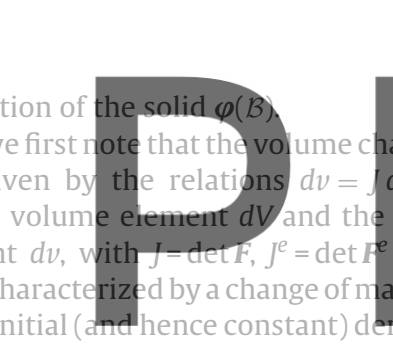

(4) $\quad \theta=\frac{d v^{g}}{d V}$ defining the current values of the varying values (varying with the deformation) of the densities $\rho_{0}$ and $\rho$ in the reference and current deformed configurations of the solid $\mathcal{B}$. The constant nature of $\rho_{0}^{\text {ini }}$ leads directly to the time evolution of the reference density $\rho_{0}$ as

$\dot{\rho}_{0}^{i n i}=0 \Rightarrow \dot{\rho}_{0}=\rho_{0}^{i n i} j^{g}=R_{0}$

for the mass source $R_{0}$ in terms of the volume change $j^{g}$ associated to the growth part of the deformation gradient $\boldsymbol{F}^{g}$. Following standard algebraic results, the mass source is then given by

$R_{0}=\rho_{0} \operatorname{tr} \hat{\boldsymbol{L}}^{g}=J^{g} \rho_{0}^{i n i} \operatorname{tr} \hat{\boldsymbol{L}}^{g}=J^{g} \rho_{0}^{i n i} \operatorname{tr}\left(\dot{\boldsymbol{F}}^{g} \cdot \boldsymbol{F}^{g^{-1}}\right)$

for the growth velocity gradient $\hat{\boldsymbol{L}}^{g}$.

In particular, isotropic growth is characterized by an isotropic growth stretch $\vartheta$ as (Lubarda and Hoger, 2002)

$\boldsymbol{F}^{g}=\vartheta \cdot \boldsymbol{I}$

so the rate of growth can be expressed as

$\hat{\boldsymbol{L}}^{g}=\dot{\boldsymbol{F}}^{g} \cdot \boldsymbol{F}^{g^{-1}}=\frac{\dot{\vartheta}}{\vartheta} \boldsymbol{I}$

resulting in a mass source Eq. (7) of the form

$R_{0}=3 \vartheta^{2} \rho_{0}^{i n i} \dot{\vartheta}$

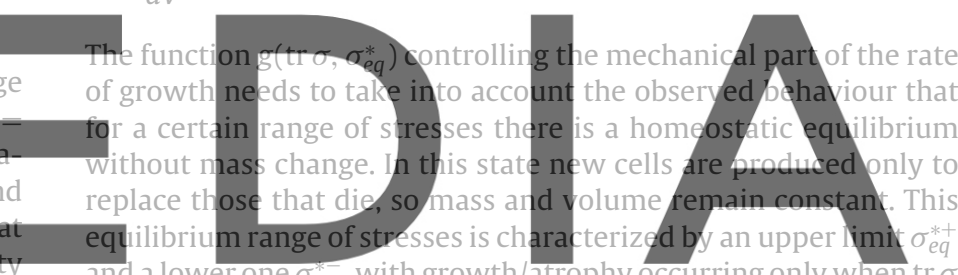
and a lower one $\sigma_{\rho q}^{*}$, with growth/atrophy occurring only when tr $\sigma$ falls outside the interval $\left(\sigma_{e q}^{*-}, \sigma_{e q}^{*+}\right)$.

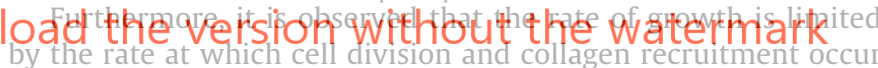
in a particular tissue. This observation is taken into account in the model by considering a maximum rate of mass production $M_{\max }$. from Eq. (10).

$M_{\max }=\frac{\left(R_{0}\right)_{\max }}{\rho_{0}^{\text {ini }}}=3 \vartheta^{2} \dot{\vartheta}_{\max }$

resulting in the maximum growth stretch rate

$\dot{\vartheta}_{\max }=\frac{M_{\max }}{3 \vartheta^{2}}$

Therefore, in this equation it is possible to see that the stretching rate is going down while the growth stretch increases. Similarly, a maximum rate of tissue resorption can be observed in the case of atrophy, defining a maximum rate of mass decrease. We denote these limit values $\dot{\vartheta}_{\max }^{+}$and $\dot{\vartheta}_{\text {max }}^{-}$for growth and atrophy, respectively.

With these considerations in mind, the growth-stimulus function $g\left(\operatorname{tr} \sigma, \sigma_{e q}^{*}\right)$ assumed in this work is depicted in Fig. 1. Its general form, with three different zones (homeostatic equilibrium zone, atrophy zone and growth zone) is similar to the one proposed by Rodríguez et al. (2007). An explicit expression of the function $g\left(\operatorname{tr} \sigma, \sigma_{e q}^{*}\right)$ can be easily devised. 


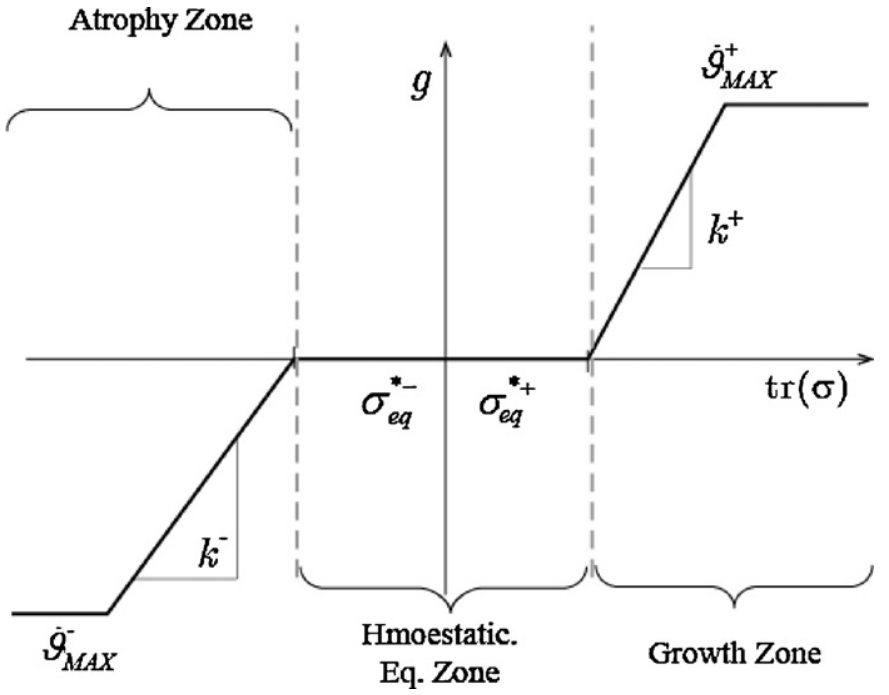

Fig. 1. General form of the mechanical growth-stimulus function.

\section{Finally, the function}

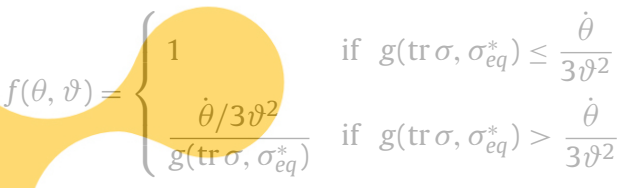

is assumed in Eq. (7) to represent the effect of the biological availability.

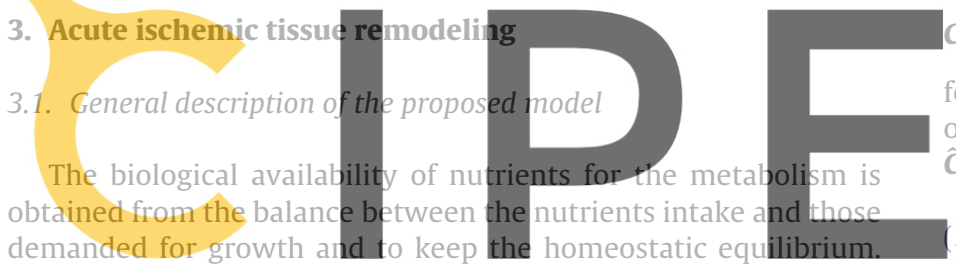

The amount of nutrients (a term we use to refer generically to oxygen, proteins, enzymes, growth factors, etc..) and metabolic waste

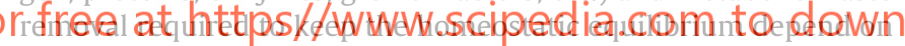
the tissue type and its function. This fact is considered in this work by means of the introduction of a non-dimensional homeostatic intake parameter named $H_{k}$. This proposed parameter accounts for the tissue daily demand of nutrients, a term that we shall use henceforth to refer to both actual nutrients as described above and any needed metabolic waste removal.

The local flux of nutrients into the metabolism is considered in this work by the function $N(t)$. See Remark 1 for the particular nutrient intake considered in the numerical examples presented in this paper. The balance between the nutrients income and the homeostatic intake at a given time, determinate the neat flux of nutrients $\dot{\theta}$ as follows

$\dot{\theta}=\dot{N}-\dot{H}_{k}$

A positive neat flux generates an excess of nutrients that become available to the metabolism. This reserve of "free" nutrients determinates the bioavailability for metabolic process, i.e. tissue growth.

If this balance is negative a critical shortage of nutrients occurs and part of the tissue undergoes a necrotic process that will be dependent on the severity of the ischemia and its duration. To address this necrotic process an evolutive a two-phase continuum theory using a multiplicative decomposition of the deformation gradient for each phase and an evolving natural configuration for the whole body is proposed. The tissue is considered as a mix of normal and necrotic tissue working in parallel each one with its own mechanical properties. The potential $W$ for the tissue model is given by:

$\rho_{0} W=k_{h} \rho_{0 h} W_{h}+k_{n} \rho_{0 n} W_{n}$

where $W_{h}$ and $W_{n}$ are the potentials of the normal (or healthy) and necrotic tissue, respectively, with the reference densities, $\rho_{0 h}$ and $\rho_{0 n}$, and the (reference) volume fractions, $k_{h}$ and $k_{n}$, of the normal and necrotic tissues, respectively. There is not enough experimental information regarding the mechanical interaction between the constituents of a tissue undergoing a necrotic process to quantify its effects. Consequently their interaction is not considered in this first attempts to study the phenomena.

The volume fractions of the healthy and necrotic parts of the tissue, in the reference configuration, are expressed as

$k_{h}=\frac{d V_{h}}{d V_{0}} \quad$ and $\quad k_{n}=\frac{d V_{n}}{d V_{0}}$

where $V_{0}$ is the total volume and $V_{h}, V_{n}$ are the healthy and necrotic tissue volumes in the reference configuration. We have $d V_{0}=d V_{h}+d V_{n}$, so $k_{h}+k_{n}=1$.

The hypothesis of the classic rule of mixtures regarding the components strain, establishes that each component is subjected to the same deformation field, leading to the following closure equation:

$\boldsymbol{F}=(\boldsymbol{F})_{h}=\boldsymbol{F}_{n}$

for the deformation gradient $\boldsymbol{F}$ of the whole tissue, and the deformation gradients $(\boldsymbol{F})_{h}$ and $(\boldsymbol{F})_{n}$ for the healthy and necrotic parts. The multiplicative decomposition (1) of the common deformation gradient $\boldsymbol{F}$ applies separately for each parts of the tissue. For the isotropic growth of interest, as defined by Eq. (8), we can write
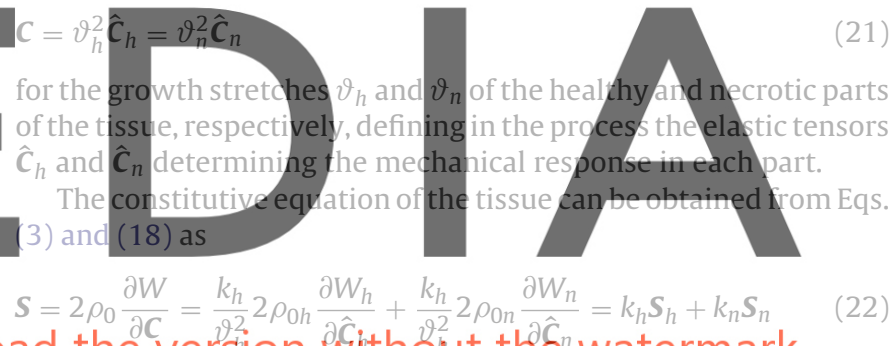

$S=2 \rho_{0} \frac{\partial W}{\partial \boldsymbol{C}}=\frac{k_{h}}{\vartheta^{2}} 2 \rho_{0 h} \frac{\partial W_{h}}{\partial \hat{\boldsymbol{C}}}+\frac{k_{h}}{\vartheta^{2}} 2 \rho_{0 n} \frac{\partial W_{n}}{\partial \hat{\boldsymbol{C}}_{n}}=k_{h} \boldsymbol{S}_{h}+k_{n} \boldsymbol{S}_{n}$

\section{with the individual stress tensors}

$\widehat{\boldsymbol{S}}_{h}=2 \rho_{0 h} \frac{\partial W_{h}}{\partial \hat{\boldsymbol{C}}_{h}}=\vartheta_{h}^{2} \boldsymbol{S}_{h}$ and $\hat{\boldsymbol{S}}_{n}=2 \rho_{0 n} \frac{\partial W_{n}}{\partial \hat{\boldsymbol{C}}_{n}}=\vartheta_{n}^{2} \boldsymbol{S}_{n}$

for the healthy and the necrotic parts of the tissue, respectively. It is important to note, as observed in Section 2 when discussing Eq. (2) that the partial derivatives in Eqs. (22) and (23) involve the mechanical deformation only, that is, they consider constant reference densities. The Cauchy stress, and in particular its trace defining the mechanical growth stimulus, is obtained from the second Piola-Kirchhoff stress $\boldsymbol{S}$ in Eq. (22) by relation (4).

Tissue necrosis affects not only the mechanical properties of the tissue but also the capability of the tissue to undergo adaptative processes. The necrotic part of the tissue is no longer an active material, that is,

$\vartheta_{n}=1$ identically(i.e. $\dot{\vartheta}_{n}=0$ )

and therefore, only the healthy part can undergo stress driven growth. This effect generates a coupling between the necrotic remodeling and the stress driven growth previously discussed. Consequently the evolution of the internal growth stretch variable for partially necrotic tissue is computed as follows

$\dot{\vartheta}_{h}=\dot{\vartheta} \sqrt[3]{k_{h}}$ 


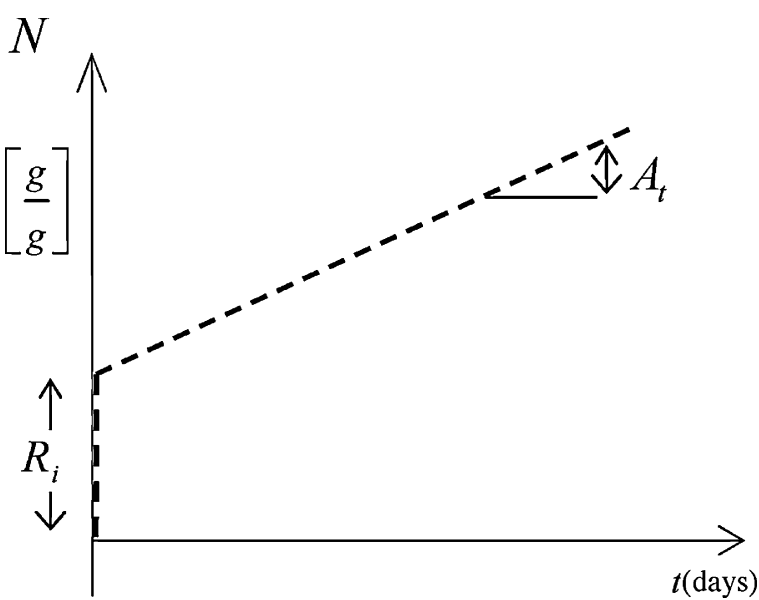

Fig. 2. Nutrients entering the system.

for the total rate $\dot{\vartheta}$ given by the considerations in Section 2 (i.e. Eq. (12) in particular).

The remodeling from healthy tissue to scar tissue takes some time, during which the mechanical properties change gradually until the tissue reaches its final configuration (Fomovsky and Holmes, 2010). The values of the mechanical properties (Eq. (18)) corresponding to that final configuration of the scar tissue are used in $W_{n}$, while for $W_{h}$ the mechanical properties of the healthy tissue are used. The temporal evolution of the necrotic tissue is controlled by means of the volume ratio $k_{n}$, which is calibrated so that the properties of the tissue mixture match the ones corresponding to experimental data. Consequently, to address this temporal evolution, a function $f n\left(t_{n}\right)$ is introd ischemic episode. The other cess is the ischemia severity evolution rule for the necrotic $k_{n}=\frac{\dot{\theta}}{\dot{H}} f n\left(t_{n}\right)$

where the ratio between the neat flux and the demand $\dot{\theta} / \dot{H}$ determinates the severity of the lack of nutrients. The healthy tissue

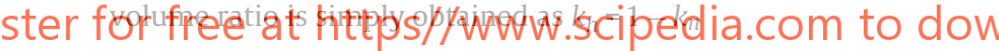

Remark 1. The amount of nutrients entering the system is taken into account by the function $N(t)$ in Eq. (17). The numerical examples presented in Section 4 consider an initial reserve of nutrients $R_{i}$ and a discrete contribution of nutrients $A_{t}$ at regular time intervals. The values of $N(t)$ are dimensionless and represent the mass increment of nutrients referred to the initial mass of the system. For instance, a value of $N=0.02$ represents the entry to the system of nutrients enough to generate an increase of tissue mass of $2 \%$ respect to its original mass. Fig. 2 shows the function of nutrients contribution.

\subsection{Application to rat myocardial infarction}

Sustained acute ischemia in cardiac tissue leads to the tissue necrosis. Because of the importance of this process several experimental studies using animal models were developed (Sunagawa et al., 1983; Lerman et al., 1983; Pfeffer et al., 1991; McCormick et al., 1994; Holmes and Covell, 1996). However, the first study that considers simultaneously the temporal evolution of scar structure, scar mechanics, and left ventricular (LV) function in large anterior myocardial infarcts in rats was published in 2010 by Fomovsky and Holmes. In their work the evolution of the mechanical properties of the scar tissue was obtained from biaxial mechanical testing at 1 , 2,3 , and 6 weeks. They also reported that infarcts in the rats were

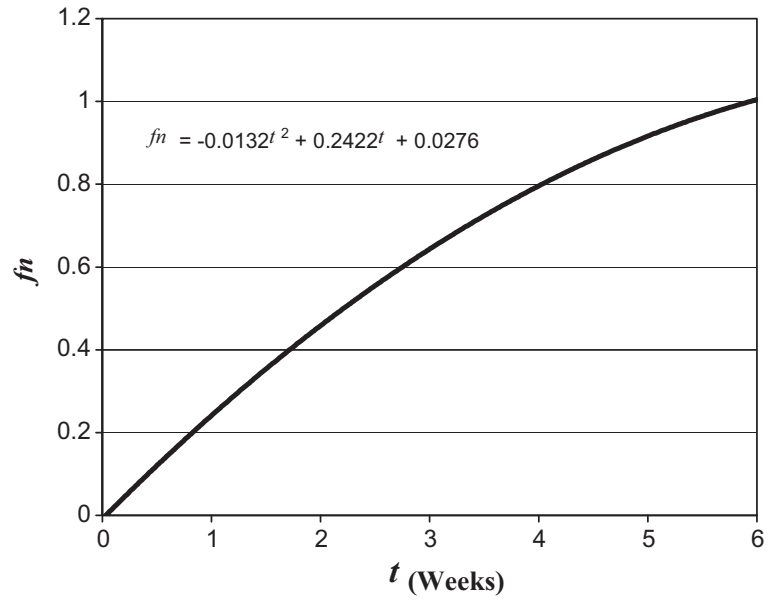

Fig. 3. Temporal evolution function $f n$ for myocardial infarcted rat tissue.

structurally and mechanically isotropic at all time points. The material was represented using a quadratic form of the energy function $W\left(I_{C}\right)$ as follows,

$W=c_{1}\left(I_{C}-3\right)^{2}$

where $c_{1}$ is a material parameter $I_{C}=\operatorname{tr}(\hat{\boldsymbol{C}})$ is the first invariant of the right Cauchy. The material parameters obtained from the mechanical testing for each time point are also provided.

The experimental data from Fomovsky and Holmes (2010) have been used to calibrate the model proposed in this work. For the fully necrotic tissue the material parameter value corresponding to the mechanical properties of the 6 th week scar $\left(c_{1}\right)_{n}=995.2 \mathrm{kPa}$ has been adopted, and for healthy tissue a value of $\left(c_{1}\right)_{h}=225.3 \mathrm{kPa}$ has been adopted. The behaviour of healthy tissue at tme 0 is recovered adopting $k_{h}=1, k_{n}=0$ and $f n=0$, while the behaviour of the 6 th week scar tissue is obtained adopting $k_{n}=0, k_{n}=1, f r=1$. To clalib ate the tempora during the $=1$. In this case the volume fitactions $k_{m}$ and $k_{h}$ are exclusively functions of $f n$. The temporal function $f n$ can then be calibrated straightforwardly fitting the mechanical properties of the proposed

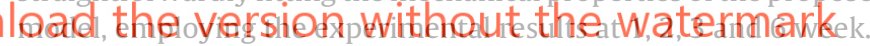

The resulting function $f n$ obtained fitting Fomovsky and Holmes (2010) data is shown in Fig. 3.

The values of the material parameters used to calibrate $f n$ are the mean values of several myocardial infarcted rat samples, therefore this function can be considered representative for this particular tissue.

\section{Representative numerical examples}

\subsection{Single element under uniaxial stress}

The application examples were performed using a 3D displacement-pressure mixed FEM in a Fortran Code. Some details of the finite element implementation considered in this work, including details on the treatment of the quasi-incompressibility of the model under consideration, can be found in Appendix A.

To assess the capability of the proposed model to represent the temporal evolution of the mechanical properties of tissue subjected to acute ischemia, a uniaxial stretching test on a single hexahedral finite element is carried out at $0,1,2,3$ and 6 weeks. A null income of nutrients is considered, consistently with the conditions imposed in Fomovsky and Holmes (2010) for the infarcted tissue. Fig. 4 shows the element, its boundary conditions and the prescribed uniaxial stretching. 


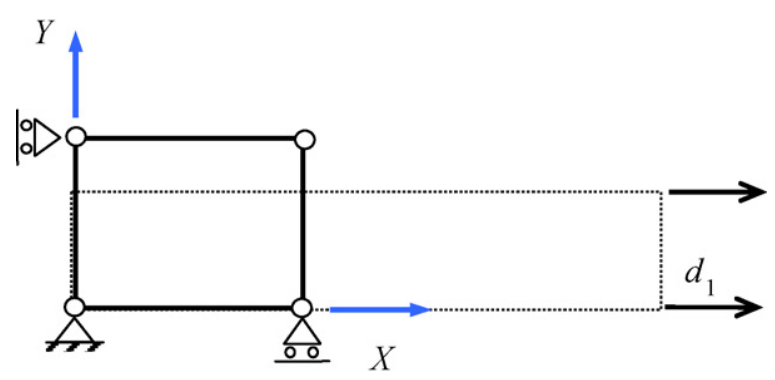

Fig. 4. Boundary conditions and prescribed displacements.

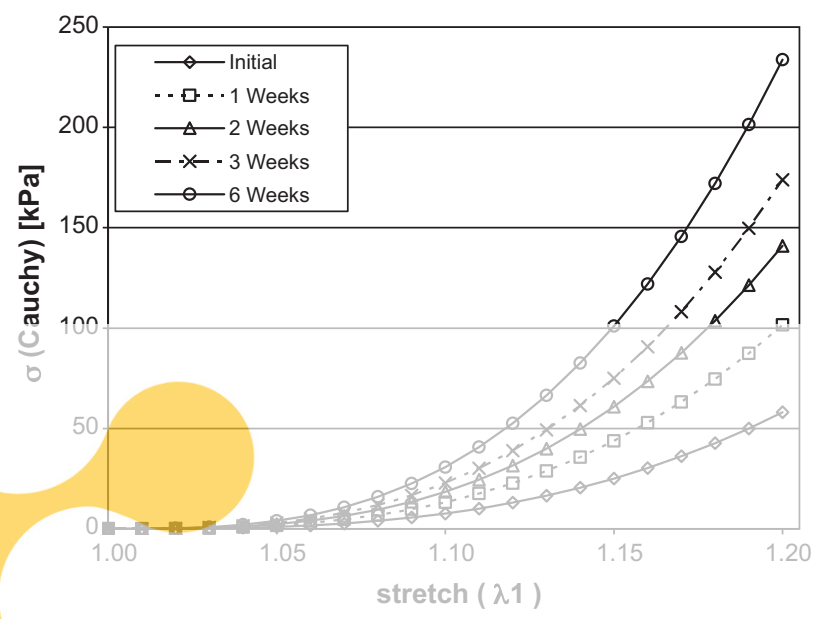

Fig. 5. Evolution of the mechanical properties in the tissue under acute ischemia.

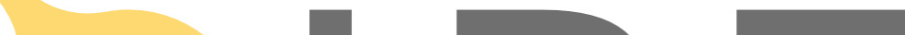

The potential for the $\mathrm{m}$ from Eq. (27) and the sarne used in the previous section the necrotic and healthy ti temporal function corresponding to myocardial infarct tissue $f n$ depicted in Fig. 3 is used.

The obtained results are shown in Fig. 5.

ster for fredęa the necrotic tissue increases and, consequently, the whole tissue response becomes stiffer. As can be seen in Fig. 5 the change in the mechanical properties is very noticeable.

\subsection{Acute ischemia in tissue patch}

The change in the mechanical properties of the acutely ischemic tissue induces an alteration at the stress and strain fields of the surrounding tissue. To study this effect, local acute ischemia is simulated in part of a tissue patch, prescribing a variable profile of the nutrients income function parameter $A_{t}$ as shown in Fig. 6a. For the sake of simplicity the stress driven growth phenomena is not considered in this example, leaving it for the example studied in the next section.

The patch dimensions are $25 \mathrm{~mm}$ long, $15 \mathrm{~mm}$ wide and $1.5 \mathrm{~mm}$ thick. The general patch response and, particularly, the behaviours of four reference points (Fig. 6b) are considered. The values of the material parameters are $\left(c_{1}\right)_{n}=995.2 \mathrm{kPa}$ and $\left(c_{1}\right)_{h}=225.3 \mathrm{kPa}$ for necrotic and healthy tissue, respectively. The temporal evolution function $f n$ from Fig. 3 is also adopted. The tissue homeostatic daily intake value is $H_{k}=0.01$, matching the nutrients income function in the patch, except in the necrotic zones with restricted nutrient income as shown in Fig. 6a. An initial nutrients reserve $R_{i}=0.015$ is considered.
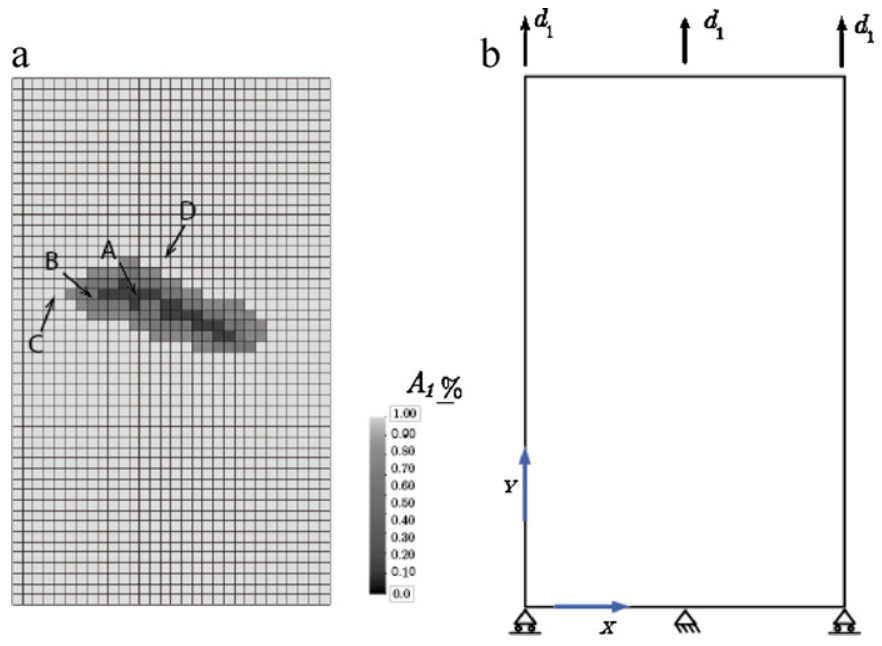

Fig. 6. (a) Nutrient income distribution along the patch and reference points location (A, B, C, D). (b) Boundary conditions and prescribed displacements.

A uniform displacement of $1.0 \mathrm{~mm}$ is applied in the upper side of the patch.

The evolution of the necrotic tissue volume fraction and the Cauchy stress trace are shown in Fig. 7 and the values for the reference points are plotted in Fig. 8.

The scar tissue is stiffer than the healthy one, in rat myocardial infarct this is a consequence mainly of the increase of the collagen content. This progressive evolution of the mechanical properties of the scar tissue along of the 6 weeks period generates an important alteration of the stress field as can be seen in Fig. 7b, where zones of stresses concentration and relaxation are clearly visible.

The aculte ischemia condition starts after 1.5 days when the zone of tissue with limited nutrients income has already consumed its initial reserve $R_{i}$ and the nutrients income is maller than the homeostatic intake. In the tissue subjected to acyte ischemia the necrotic tissue fraction increases over time, being this ffect more noticeable near to the scar boundary, shows a smaller variation while point $D$, located outside the scar, shows no remodeling as expected (Fig. 8b).

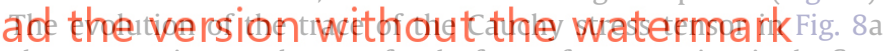
shows superimposed curves for the four reference points in the first day period coincidently with the application of the patch stretch. As the remodeling process progress the stress field is no longer uniform and the curves diverge, the plot shows an important variation of the stresses in the reference points.

\subsection{Necrosis and stress driven growth interaction}

This final example considers the both mentioned processes, stress driven growth and necrotic tissue remodeling. As seen in the previous example the necrosis alters the stress field of the scar tissue and the healthy surrounding tissue. This concentration of supra-homeostatic stress in zones of the surrounding tissue that have a sustained flux of nutrients, can lead to a stress driven growth process. To address this interaction the same mesh, boundary conditions and material properties from example in Section 4.1 are considered and tissue growth is also enabled. To allow growth the values of $A_{t}$ are increased a $10 \%$ outside the zone of limited nutrients income. The maximum mass production adopted is $R_{\max }=0.13 \%$ day and the growth upper limit is $\sigma_{e q}^{*+}=1.05 \mathrm{kPa}$. The results are shown in Figs. 9 and 10.

The concentration of stresses in the healthy tissue evolves along the time while the scar tissue assumes its final structure. This mechanical stimulus induces a simultaneous growth process of the 
a

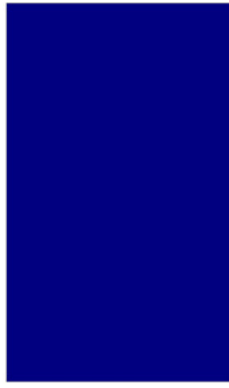

b

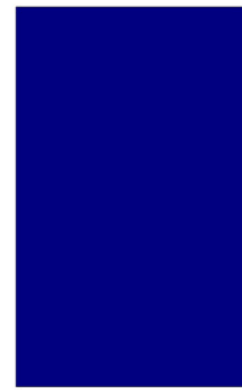

$t=0$
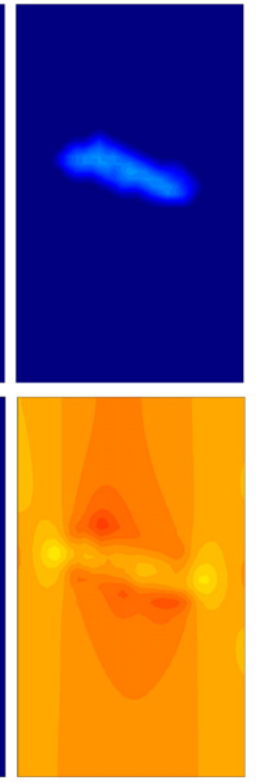

$t=10$
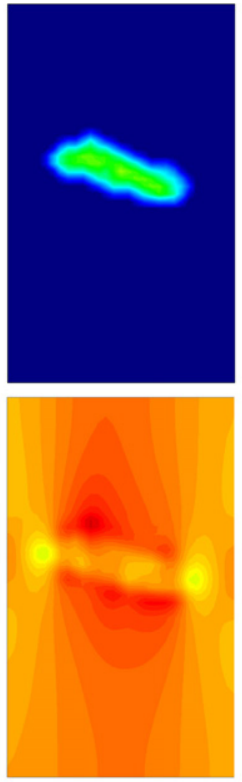

$t=20$
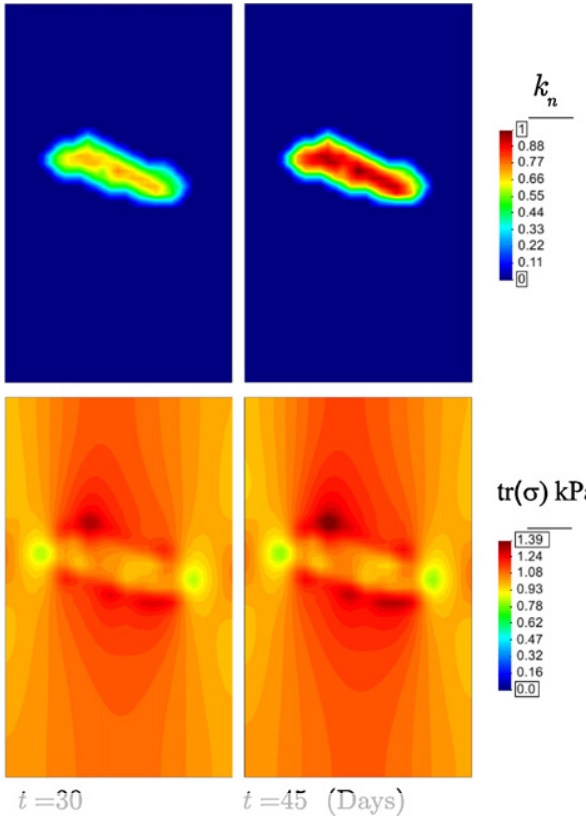

$\operatorname{tr}(\sigma) \mathrm{kPa}$

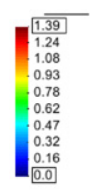

Fig. 7. (a) $k_{n}$ evolution. (b) Cauchy stress trace evolution.

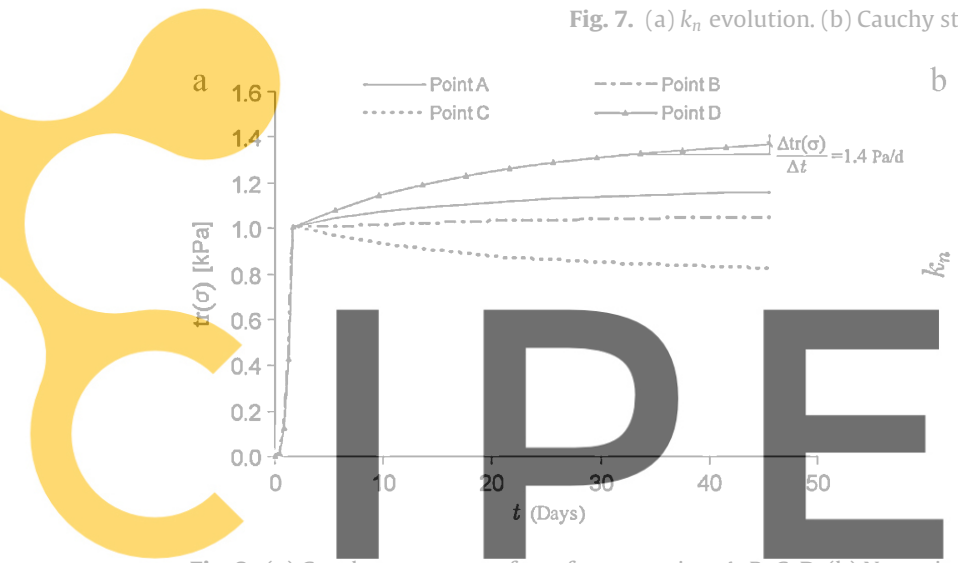

Fig. 8. (a) Cauchy stress trace for reference points A, B, C, D. (b) Necrotic

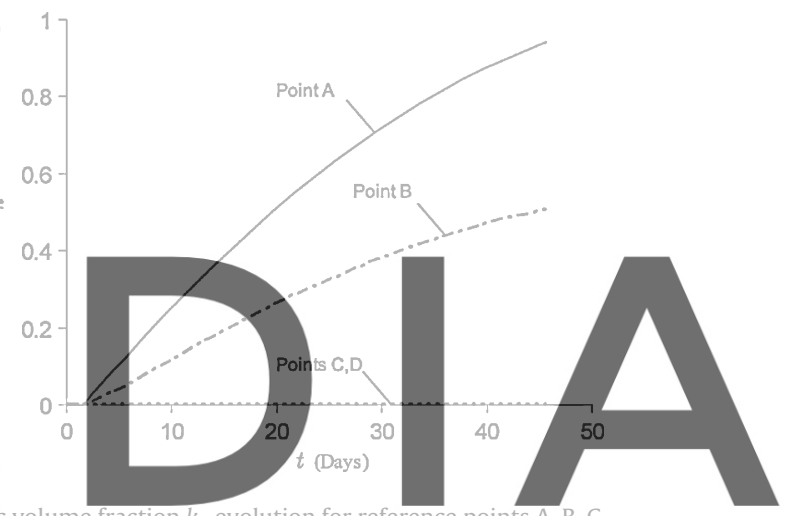

ster for free at hittps//www.scipedia.com to download the version without the watermark
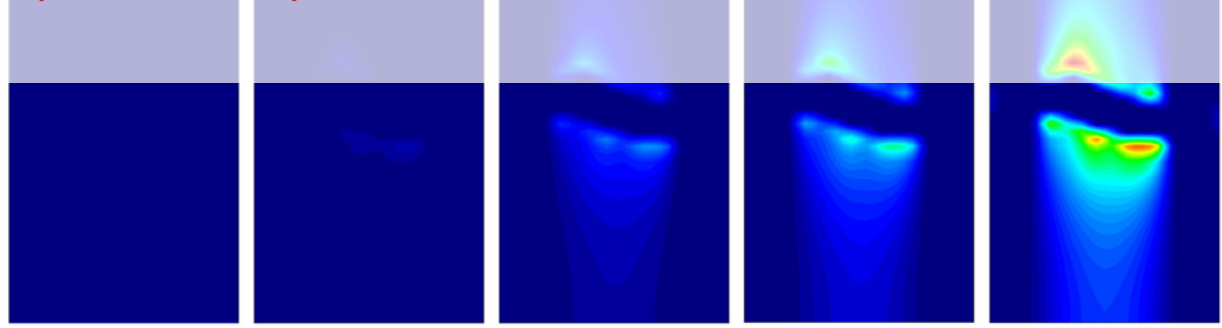

$$
\frac{\vartheta}{-10}
$$
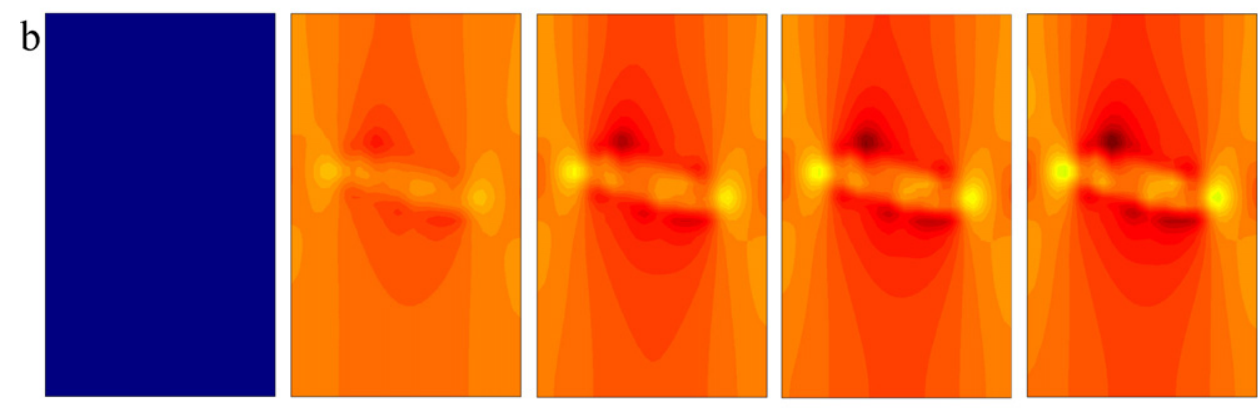

$\operatorname{tr}(\sigma) \mathrm{kPa}$

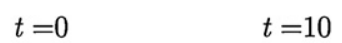

$t=20$

$t=30$

$t=45$ (Days)

Fig. 9. (a) Growth stretch evolution. (b) Cauchy stress trace evolution during growth. 

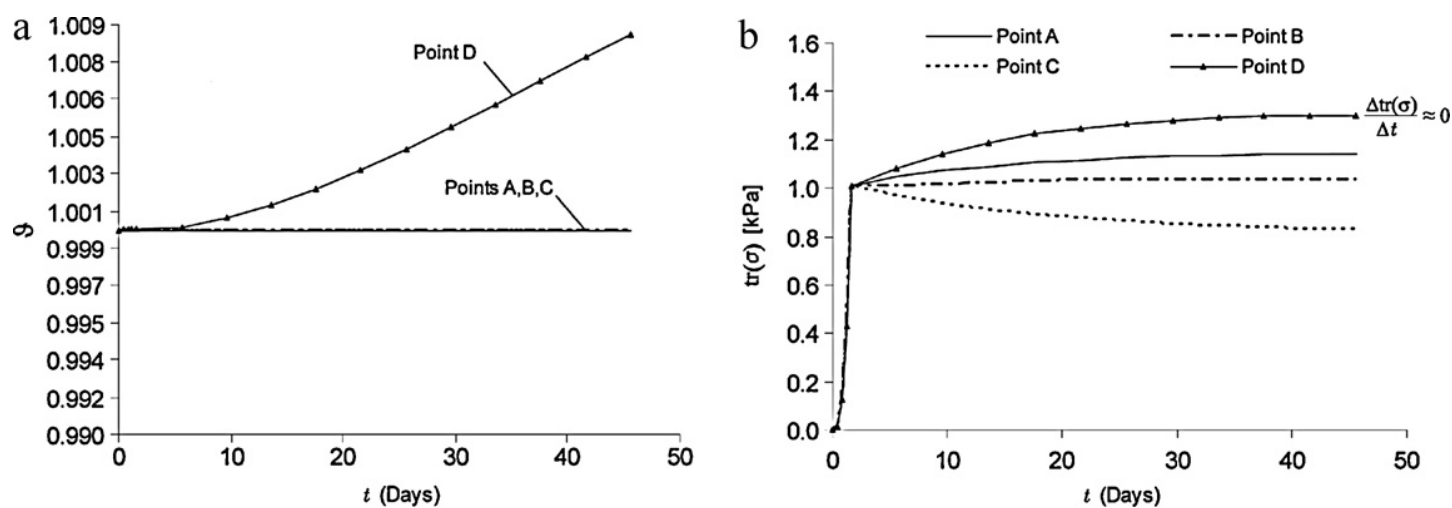

Fig. 10. (a) Cauchy stress trace for reference points A, B, C, D. (b) Growth stretch $\vartheta$ evolution for reference points A, B, C, D.

healthy tissue zones with supra-homeostatic values of the Cauchy stress trace as can be seen in Fig. 9a. Also it can be seen that tissue undergoing the necrotic process has an increased stress field (Figs. 9b and 10b), but since it lacks any free nutrients the metabolic field does not enable the growth process and no growth stretch is developed in the necrotic tissue area (Figs. 9a and 10a).

Tissue growth induces a stress relaxation as can be noted by comparing Cauchy stress trace slope in Figs. 8 and 10, for reference point D.

\section{Conclusions}

A model for the numerical simulation of the necrosis process and stress driven growth is proposed in this work. The necrotic remodeling phenomena is treated considering a reinterpretation the evolution of the volui model capabilities have tion examples regarding to the model can be applied tr

The model is able between growth and acute ischessily capture the interaction biological and mechanical fields. where

$J=\operatorname{det}[\boldsymbol{F}]=\operatorname{det}\left[\boldsymbol{F}_{\mathrm{vol}}\right], \quad \operatorname{det}[\overline{\boldsymbol{F}}]=1$

The multiplicative split of the deformation gradient leads to an additive strain energy decomposition

$W=U(J)+\bar{W}(\bar{C})$

where $\overline{\boldsymbol{C}}$ is the deviatoric part of the right Cauchy-Green tensor, $\overline{\boldsymbol{C}}=\overline{\boldsymbol{F}}^{T} \overline{\boldsymbol{F}}$.

This uncoupled energy function leads to an uncoupled stress-strain relationship. The volumetric strain is associated, in the reference configuration with a hydrostatic pressure term $p$, given by:

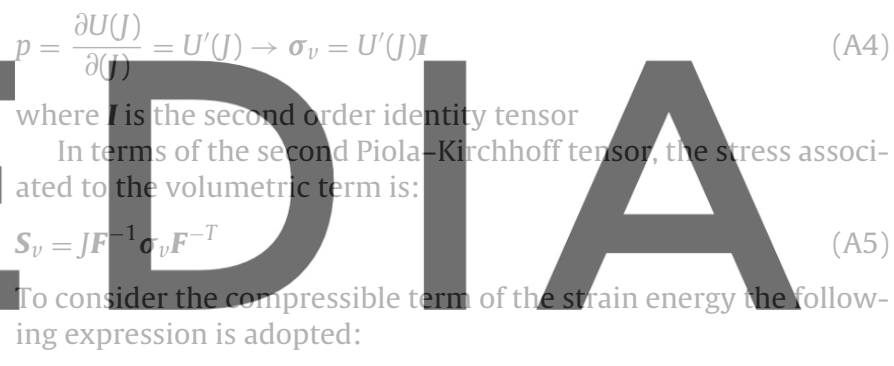

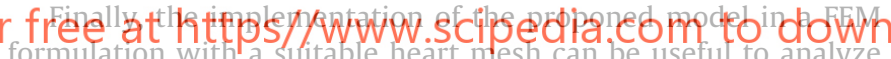

the change in the functional capabilities in acute ischemic cases.

\section{Acknowledgments}

S. Oller, L. Nallim and F. Bellomo acknowledge the support of projects CAMBIO (MAT2009-10258), Ministerio de Ciencia e Innovación of Spain; AECID (A/024063/09); and by CIMNE, Spain. F. Armero acknowledges the financial support of the AFOSR under Grant no. FA9550-08-1-0410 with UC Berkeley, USA, and of the AGAUR of the Generalitat de Catalunya, Spain, under the PIV 2010 program with grant $\mathrm{N}^{\circ}$. 2010 PIV00142.

\section{Appendix A.}

\section{A.1. Soft tissue incompressibility}

The treatment of the incompressibility in the material model follows closely the approach developed in Crisfield (1997). To this end the volumetric $\left(\boldsymbol{F}_{\mathrm{vol}}\right)$ and deviatoric $(\overline{\boldsymbol{F}})$, terms of the deformation gradient are split as follows

$\boldsymbol{F}=\boldsymbol{F}_{\mathrm{vol}} \overline{\boldsymbol{F}}, \quad \boldsymbol{F}_{\mathrm{vol}}=J^{1 / 3} \boldsymbol{I}, \quad \overline{\boldsymbol{F}}=J^{1 / 3} \boldsymbol{F}$

oad) the 1 koversion without the watermark

where $k$ is the bulk modulus of the material and the incompressible form can be recovered by letting $k$ tend to infinity. This choice for the volumetric function $U(J)$ allows a simple treatment in the penalty formulation considered here, taking into account that the Jacobian $J$ stays close to 1 in the quasi-incompressible problem of interest. After this consideration the volumetric part of the stress tensor results:

$\boldsymbol{S}_{v}=J k(J-1) \boldsymbol{C}^{-1}$

The volumetric term of the Cauchy stress tensor $\boldsymbol{p}=\boldsymbol{\sigma}_{v}$ in the reference configuration results:

$\boldsymbol{p}=-k(J-1) \boldsymbol{I}$

and the deviatoric part of the stress is given by:

$\overline{\boldsymbol{S}}=2 \frac{\partial \bar{W}(\overline{\boldsymbol{C}})}{\partial \boldsymbol{C}}$

The expression of the stress is obtained by adding the deviatoric and volumetric terms as follows:

$\boldsymbol{S}=2 \frac{\partial \bar{W}(\overline{\boldsymbol{C}})}{\partial \boldsymbol{C}}-J k(J-1) \boldsymbol{C}^{-1}$

This form of the strain energy is convenient for its implementation in a mixed pressure displacement FEM code, where nodal values of 
$\boldsymbol{p}$ are used as Lagrange multipliers to enforce the incompressibility constraint.

\section{A.2. Mixed displacement-pressure formulation}

In this work a two field formulation is used, pressure and displacement are considered as variables at the structural level following Crisfield (1997). To this end different shape functions for the displacement and pressure variables, with the latter taking a lower order variation, are employed,

$p=\sum h p_{i} p_{i} \quad u=\sum h_{i} u_{i} \quad v=\sum h_{i} v_{i} \quad w=\sum h_{i} w_{i}$

where $p_{i}$ are the nodal pressure variables and $u, v, w$ are the nodal displacement variables.

Eq. (A11) can be written as:

$p=\boldsymbol{h}_{p}^{T} \hat{\boldsymbol{p}}$

where $\boldsymbol{h}_{p}=\left[h_{p 1}, h_{p 2}, \ldots, h_{p n}\right]$ and $\hat{\boldsymbol{p}}=\left[p_{1}, p_{2}, \ldots, p_{n,}\right]$

Virtual work can be used to find the out-of balance force vector, g where

$g=q_{i}-q_{e}=\int B_{n l}^{T} S d V_{0}-q_{e}$

(A13)

with $\boldsymbol{S}$ as the second Piola-Kirchhoff stresses, $\boldsymbol{B}_{n l}$ relates displacements and strains, $\boldsymbol{q}_{i}, \boldsymbol{q}_{e}$ are the internal and external forces vectors, respectively.

Assuming exact equilibrium form a previous increment differentiation of (A13) leads to:

\section{$\delta \boldsymbol{q}_{e}=\delta \boldsymbol{q}_{i}=\int \boldsymbol{B}_{n l}^{T} \delta \boldsymbol{S} d V_{0}+\int \delta \boldsymbol{B}_{n l}^{T} S d V_{0}$}

(A14)
Substituting Eq. (A22) into Eq. (A21)

$\boldsymbol{a}=\boldsymbol{P} \delta \hat{\boldsymbol{p}}=\int \boldsymbol{B}_{n l}^{T} \mathbf{g}^{k} \boldsymbol{h}_{p}^{T} d V_{0} \delta \hat{\boldsymbol{p}}$

from which:

$\boldsymbol{P}_{u p}=\frac{\partial q_{i}}{\partial \hat{\boldsymbol{p}}}=\int \boldsymbol{B}_{n l}^{T} \boldsymbol{g}^{k} \boldsymbol{h}_{p}^{T} d V_{0}$

To apply the pressure-displacement relationship from Eq. (A8) Galerkin method is applied to obtain a "weak form" of this equation and multiplying by $\delta p$ and integrating over the element the following expression is obtained:

$$
\begin{aligned}
-\int\left((J-1)+\frac{1}{k} p\right) \delta p d V_{0} & =-\delta \hat{\boldsymbol{p}}^{T} \int \boldsymbol{h}_{p}\left((J-1)+\frac{1}{k} p\right) d V_{0} \\
& =\delta \hat{\boldsymbol{p}}^{T} \boldsymbol{f}=0
\end{aligned}
$$

This relationship must hold for any arbitrary pressure variation so that with $\boldsymbol{f}$ representing the lack of pressure compatibility:

$\left.\boldsymbol{f}=-\int \boldsymbol{h}_{p}(J-1)+\frac{1}{k} p\right) d V_{0}=0$

Assuming continued satisfaction of Eq. (A26) from an "equilibrium state":

$\delta \boldsymbol{f}=\frac{\partial \boldsymbol{f}}{\partial \boldsymbol{u}} \delta \boldsymbol{u}+\frac{\partial \boldsymbol{f}}{\partial \hat{\boldsymbol{p}}} \delta \hat{\boldsymbol{p}}=\boldsymbol{P}_{u p}^{T} \delta \boldsymbol{u}+\boldsymbol{H} \delta \hat{\boldsymbol{p}}=0$

(A27)

where $\boldsymbol{P}_{\text {up }}$ has already been defined in Eq. (A24) and $\boldsymbol{H}$ is given by: $\delta \boldsymbol{S}_{i j}=\mathbb{C}_{i j k l} \delta \boldsymbol{E}_{k l}+\boldsymbol{G}_{i j} \delta u$

where $\mathbb{C}_{i j k l}$ is a fourth order

by:
Considering the stresses differentiation:

$G_{i j}=-I_{3}^{-1 / 2} C_{i j}^{-1}$

Eq. (A15) can be written in matrix form as:
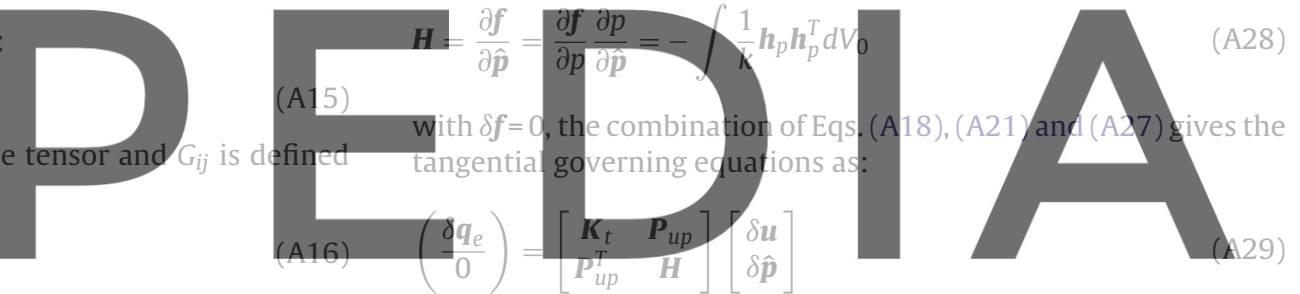

where $\mathbb{C}^{S}$ is a matrix and $g^{k}$ a column vector. Substituting Eq. (A17) in Eq. (A14) and considering that $\delta E=\boldsymbol{B}_{n l}(\hat{\boldsymbol{p}}) \delta \boldsymbol{u}$, the following expression is obtained:

$\delta \boldsymbol{q}_{e}=\frac{\partial \mathbf{q}_{i}}{\partial \boldsymbol{u}} \delta \boldsymbol{u}=\boldsymbol{K}_{t} \delta \boldsymbol{u}+\boldsymbol{a}$

where

$\boldsymbol{K}_{t}=\frac{\partial \boldsymbol{q}_{i}}{\partial \boldsymbol{u}}=\int \boldsymbol{B}_{n l}^{T} C^{S} \boldsymbol{B}_{n l} d V_{0}+\int \boldsymbol{G}^{T} \hat{\boldsymbol{S}} d V_{0}$

$\hat{\boldsymbol{S}}$ contains the second Piola-Kirchhoff tensor stresses, and is given by:

$\hat{\boldsymbol{S}}=\left[\begin{array}{lll}\overline{\boldsymbol{S}} & 0 & 0 \\ 0 & \overline{\boldsymbol{S}} & 0 \\ 0 & 0 & \overline{\boldsymbol{S}}\end{array}\right], \quad \operatorname{con} \overline{\boldsymbol{S}}=\left[\begin{array}{lll}\boldsymbol{S}_{11} & \boldsymbol{S}_{12} & \boldsymbol{S}_{13} \\ \boldsymbol{S}_{21} & \boldsymbol{S}_{22} & \boldsymbol{S}_{23} \\ \boldsymbol{S}_{31} & \boldsymbol{S}_{32} & \boldsymbol{S}_{33}\end{array}\right]$

The pressure coupling vector $\boldsymbol{a}$ in Eq. (A18) is given by:

$\boldsymbol{a}=\int \boldsymbol{B}_{n l}^{T} \mathbf{g}^{k} d V_{0} \delta p=\boldsymbol{P} \delta \hat{\boldsymbol{p}}$

Pressure change $\delta p$ in terms of its nodal variables is given by:

$\delta p=\boldsymbol{h}_{p}^{T} \delta \hat{\boldsymbol{p}}$

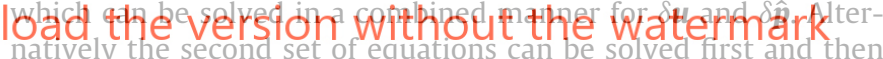
substituted into the first set to give:

$\delta \boldsymbol{q}_{e}=\left[\boldsymbol{K}_{t}-\boldsymbol{P}_{u p} \boldsymbol{H}^{-1} \boldsymbol{P}_{u p}^{T}\right] \delta \boldsymbol{u}=\overline{\boldsymbol{K}}_{t} \delta \boldsymbol{u}$

where $\overline{\mathbf{K}}_{t}$ is the effective tangent stiffness matrix.

To form the basis of a Newton-Raphson iteration we expand Eqs. (A13) and (A29) using truncated Taylor series. Hence from Eq. (A13) we have:

$\mathbf{g}_{n}=0=\mathbf{g}_{0}+\frac{\partial \mathbf{g}}{\partial \boldsymbol{u}} \delta \boldsymbol{u}+\frac{\partial \mathbf{g}}{\partial \hat{\boldsymbol{p}}} \delta \hat{\boldsymbol{p}}$

while from Eq. (A26) we have:

$\boldsymbol{f}_{n}=0=\boldsymbol{f}_{0}+\frac{\partial \boldsymbol{f}}{\partial \boldsymbol{u}} \delta \boldsymbol{u}+\frac{\partial \boldsymbol{f}}{\partial \hat{\boldsymbol{p}}} \delta \hat{\boldsymbol{p}}$

Dropping the subscript 0, Eqs. (A31) and (A32) can be combined to give:

$-\left(\begin{array}{c}\mathbf{g} \\ \boldsymbol{f}\end{array}\right)=\left[\begin{array}{cc}\boldsymbol{K}_{t} & \boldsymbol{P}^{u p} \\ \boldsymbol{P}_{u p}^{T} & \boldsymbol{H}\end{array}\right]\left[\begin{array}{c}\Delta \boldsymbol{u} \\ \Delta \hat{\boldsymbol{p}}\end{array}\right]$

Using a mixed formulation Eq. (A33) can be solved directly as a part of a Newton-Raphson loop. 


\section{References}

Ambrosi, D., Guana, F., 2007. Stress modulated growth. Math. Mech. Solids 12, 319-343.

Ambrosi, D., Preziosi, L., Vitale, G., 2010. The insight of mixtures theory for growth and remodeling. Z. Angew. Math. Phys. 61, 177-191.

Ateshian, G.A., 2009. On the theory of reactive mixtures for modeling biological growth. Biomech. Model. Mechanobiol. 6, 423-445.

Crisfield, M.A., 1997. Nonlinear Finite Element Analysis of Solids and Structures, Vols I, II. Wiley.

Epstein, M., Maugin, G.A., 2000. Thermomechanics of volumetric growth in uniform bodies. Int. J. Plast. 16, 951-978.

Fomovsky, G.M., Holmes, J.W., 2010. Evolution of scar structure, mechanics, and ventricular function after myocardial infarction in the rat. Am. J. Physiol. Heart Circ. Physiol. 298, H221-H228.

Goktepe, S., Abilez, O.J., Kuhl, E., 2010. A generic approach towards finite growth with examples of athlete's heart, cardiac dilation, and cardiac wall thickening. J. Mech. Phys. Solids 58, 1661-1680.

Himpel, G., Kuhl, E., Menzel, A., Steinmann, P., 2005. Computational modelling of isotropic multiplicative growth. CMES 8 (2), 119-134.

Holmes, J.W., Covell, J.W., 1996. Collagen fiber orientation in myocardial scar tissue. Cardiovasc. Pathobiol. 1, 15-22.

Humphrey, J., Rajagopal, K. A, 2002. constrained mixture model for growth and remodeling of soft tissues. Math. Models Methods Appl. Sci. 22, 407-430.

Kroon, W., Delhaas, T., Arts, T., Bovendeerd, P., 2009. Computational modeling of volumetric soft tissue growth: application to the cardiac left ventricle. Biomech. Model. Mechanobiol. 8, 309-310.
Kuhl, E., Maas, R., Himpel, G., Menzel, A., 2007. Computational modeling of arterial wall growth: attempts towards patient-specific simulations based on computer tomography. Biomech. Model. Mechanobiol. 6, 321-331.

Lerman, R.H., Apstein, C.S., Kagan, H.M., Osmers, E.L., Chichester, C.O., Vogel, W.M. Connelly, C.M., Steffee, W.P., 1983. Myocardial healing and repair after experimental infarction in the rabbit. Circ. Res. 53, 378-388.

Lubarda, V.A., Hoger, A., 2002. On the mechanics of solids with a growing mass. Int. J. Solids Struct. 39, 4627-4664.

McCormick, R.J., Musch, T.I., Bergman, B.C., Thomas, D.P., 1994. Regional differences in LV collagen accumulation and mature cross-linking after myocardial infarction in rats. Am. J. Physiol. Heart Circ. Physiol. 266, H354-H359.

Oller, S., Bellomo, F.J., Armero, F., Nallim, L.G., 2010. A stress driven growth model for soft tissue considering biological availability. In: IOP Conference Series: Materials Science and Engineering, Proceedings of WCCM/APCOM, doi:10.1088/1757-899X/10/1/012121.

Pfeffer, J.M., Pfeffer, M.A., Fletcher, P.J., Braunwald, E., 1991. Progressive ventricular remodeling in rat with myocardial infarction. Am. J. Physiol. Heart Circ. Physiol. 260, H1406-H1414.

Rodriguez, E., Hoger, A., McCulloch, A.D., 1994. Stress-dependent finite growth in soft elastic tissues. J. Biomech. 21 (4), 455-467.

Rodríguez, J., Goicolea, J.M., Gabaldón, F., 2007. A volumetric model for growth of arterial walls with arbitrary geometry and loads. J. Biomech. 40, 961-971.

Sunagawa, K., Maughan, W.L., Sagawa, K., 1983. Effect of regional ischemia on the left ventricular end-systolic pressure-volume relationship of isolated canine hearts. Circ. Res. 52, 170-178.

Taber, L.A., 2009. Towards a unified theory for morphomechanics. Philos. Trans. R. Soc. A $367,3555-3583$.
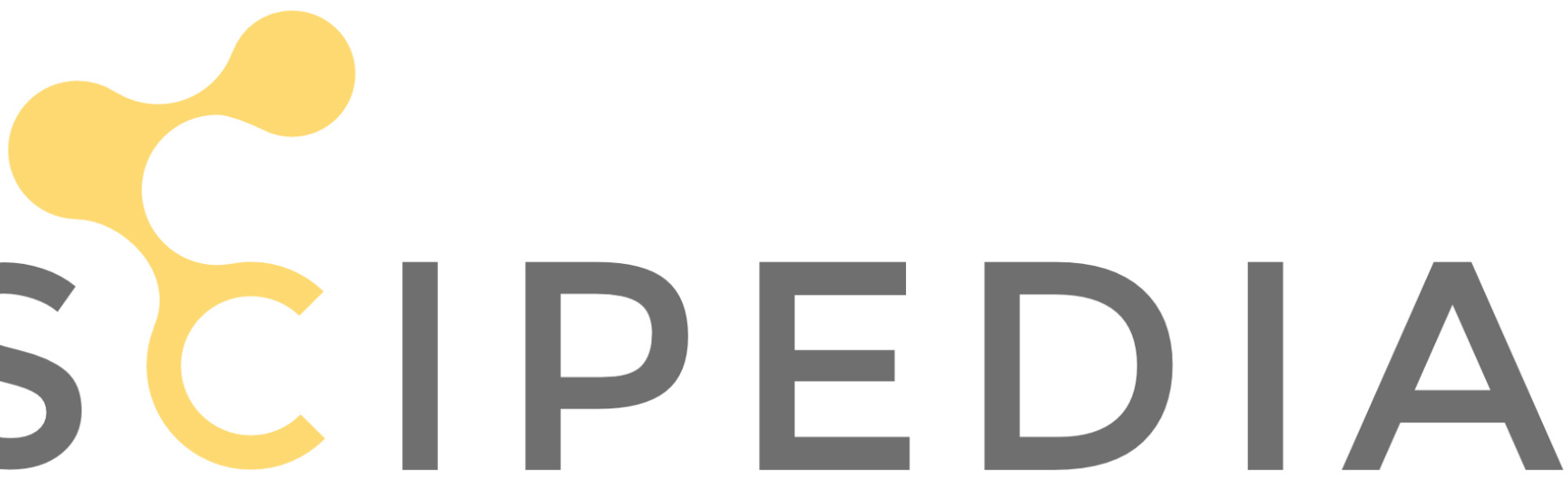